\title{
Fluctuations and Stability of Superfluid Turbulence at mK Temperatures
}

\author{
W. Schoepe \\ Institut für Experimentelle und Angewandte Physik, Universität Regensburg, D-93040 Regensburg, Germany
}

(Received 30 October 2003; published 5 March 2004)

\begin{abstract}
Turbulent flow of superfluid ${ }^{4} \mathrm{He}$ at $\mathrm{mK}$ temperatures around an oscillating microsphere is known to be unstable at low driving forces, switching intermittently between turbulent and laminar phases. The lifetimes of the turbulent phases are exponentially distributed, and the mean lifetimes grow exponentially with the square of the driving force. These experimental results are attributed to statistical fluctuations of the density $L$ of the vortex line length. As a result, a normal probability distribution of $L^{2}$ is found having a standard deviation of $2.9 \times 10^{14} \mathrm{~m}^{-4}$ and a spectral bandwidth $\Delta \omega \approx 13 \mathrm{~s}^{-1}$.

DOI: 10.1103/PhysRevLett.92.095301

PACS numbers: $67.40 . V s, 47.27 . \mathrm{Cn}$
\end{abstract}

Turbulence in superfluid ${ }^{4} \mathrm{He}$ at very low temperatures has gained considerable interest in recent years. When the normal fluid component is absent (only a very dilute gas of ballistically propagating thermal phonons is left below about $0.5 \mathrm{~K}$ ) turbulence in the pure superfluid can be studied. Because of its quantum mechanical properties, the turbulent state of the superfluid consists of a tangle of identical and individual vortex lines having a core of atomic size and the same quantized circulation $\kappa=$ $h / m_{4}$, where $h$ is Planck's constant and $m_{4}$ is the mass of a ${ }^{4} \mathrm{He}$ atom [1]. This situation appears to be much simpler than turbulence in a classical liquid, and one might wonder whether an understanding of superfluid turbulence could have an impact on the more complicated problem of classical turbulence. Furthermore, turbulence in Bose-Einstein condensed gases has many properties in common with that in the dense Bose liquid ${ }^{4} \mathrm{He}$ [2], and there is great interest in comparing both cases. In addition, turbulence studies in the fermionic superfluid ${ }^{3} \mathrm{He}$, where the powerful tool of nuclear magnetic resonance is available, very recently have yielded exciting results [3] that demonstrate both similarities and differences of the turbulent behavior when compared with ${ }^{4} \mathrm{He}$.

In contrast to superfluid ${ }^{3} \mathrm{He}$ and the condensed Bose gases, the experimental techniques for turbulence studies in ${ }^{4} \mathrm{He}$ at very low temperatures are rather limited so far, because the standard technique to detect vorticity by absorption of second sound, which works well above $1 \mathrm{~K}$, fails in the millikelvin regime. Recently, a detailed study of turbulent flow around an oscillating microsphere has been published [4]. The flow pattern (either turbulence or potential flow) determines the oscillation amplitude due to the different drag forces, and therefore it can be easily identified. The experimental results on the stability of the turbulent flow are analyzed quantitatively in this work. Based on a model of statistical fluctuations of the density of the vortex lines, an analysis is presented that gives a first insight into the probability density and the spectral distribution of these fluctuations in the $\mathrm{mK}$ regime.

Without going into the details of the experimental technique, which has been described in Ref. [4], the essential results can be summarized as follows. At low driving forces and hence at low oscillation (and velocity) amplitudes (oscillation frequency ca. $120 \mathrm{~Hz}$ ), the flow around the sphere (radius $0.12 \mathrm{~mm}$ ) is laminar, the only drag force is due to ballistic phonon scattering, and, therefore, it decreases rapidly below $0.5 \mathrm{~K}$. When the driving force is increased, an abrupt transition to turbulence takes place at a critical velocity amplitude of about $20 \mathrm{~mm} / \mathrm{s}$. However, below $0.5 \mathrm{~K}$ turbulence is observed to be unstable and the flow switches intermittently between turbulence and potential flow, indicating that the balance between vortex production and annihilation is still marginal.

In Fig. 1 two time series of the velocity amplitude at fixed temperature and driving force are shown. During a turbulent phase the velocity is low due to the large turbulent drag while during a laminar phase the velocity amplitude relaxes towards a higher level determined by phonon scattering until a new turbulent phase is born. In the following the lifetimes of the turbulent phases are discussed in detail.

Figure 2(a) shows that the lifetimes $t$ of the turbulent phases are exponentially distributed and the mean lifetime $\langle t\rangle$ can be determined. In Fig. 2(b) the mean

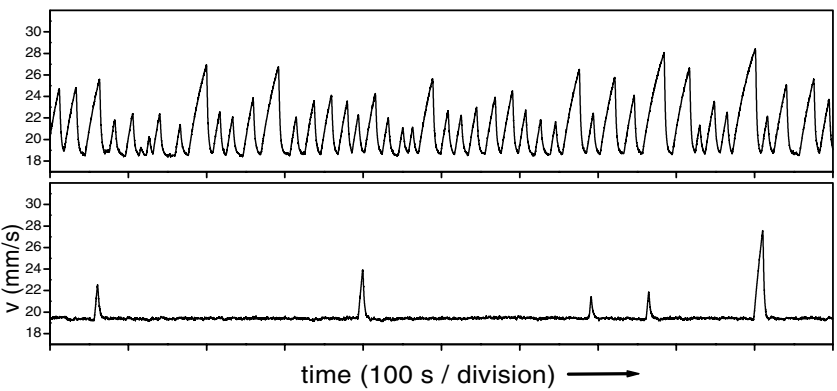

FIG. 1. Time series of intermittent switching of the velocity amplitude at $300 \mathrm{mK}$ and two different driving forces. The low level corresponds to turbulent flow while the increase occurs during a laminar phase. At a driving force of $55 \mathrm{pN}$ (upper trace) the turbulent phases are short-lived while at $75 \mathrm{pN}$ (lower trace) their lifetimes are much longer. 
lifetimes at various temperatures are displayed as a function of the turbulent drag force $F_{\text {turb }}$, which is the difference between the driving force and the linear phonon drag at the turbulent velocity [4]. It is obvious that a universal curve is obtained for all temperatures showing a very rapid increase of $\langle t\rangle$ until the lifetimes exceed the recording time and turbulence appears to be stable. The variation of the mean lifetime is described by

$$
\langle t\rangle=t_{0} \exp \left(\frac{F_{\text {turb }}}{F_{0}}\right)^{2},
$$

where $t_{0}=0.5 \mathrm{~s}$ and $F_{0}=18 \mathrm{pN}$. Multiplying the forces in Eq. (1) with the velocity amplitude of the turbulent phase and dividing by 2 , we have the dissipated power $P$ as a new variable and the exponent becomes $\left(P / P_{0}\right)^{2}$ with $P_{0}=0.20 \mathrm{pW}$. The dissipated power per unit mass $\epsilon=$ $P / M\left(M=0.46 \times 10^{-6} \mathrm{~kg}\right.$ is the mass of the turbulent liquid in the measuring cell) is an important quantity both in classical turbulence and in quantum turbulence [5] because it is related to the vorticity. Assuming that the basic ingredients in superfluid turbulence are the circulation quantum $\kappa$ and the length of vortex lines per unit volume $L$ (which has the dimension $\mathrm{m}^{-2}$ ) we have from dimensional arguments $\epsilon \sim \kappa^{3}\left\langle L^{2}\right\rangle$ and a vorticity $\kappa\langle L\rangle$. Averages are used because it is not known how the dissipated power is distributed over the volume of the turbulent liquid $\left(3.1 \times 10^{-9} \mathrm{~m}^{3}\right)$. We replace now $P / P_{0}$ by $\left\langle L^{2}\right\rangle / L_{0}^{2}$, where $L_{0}=\sqrt{P_{0} / M \kappa^{3}}=2.1 \times 10^{7} \mathrm{~m}^{-2}$ and hence Eq. (1) can be written

$$
\langle t\rangle=t_{0} \exp \left(\frac{\left\langle L^{2}\right\rangle}{L_{0}^{2}}\right)^{2} .
$$

The following model to describe these experimental results is based on statistical fluctuations of the vorticity. It is suggested that a fluctuation of $L^{2}$ below the mean value and larger than a certain threshold will cause a breakdown of the turbulent state and a recovery of the laminar phase. This idea is sketched in Fig. 3 where a random process is shown to fluctuate around its mean value. The lifetime of the turbulent state is given by the time of the first down crossing below a certain level. The properties of level crossing by a random process are mathematically very well investigated [6] and extensive use is made of this literature in what follows. First of all, the down crossings constitute a Poisson process on the time axis, and therefore the time intervals are exponentially distributed. Accordingly, the time of the first down crossing is also exponentially distributed when the system is restarted many times with a new turbulent phase. Moreover, it is plausible that an increased drive will shift the mean value $\left\langle L^{2}\right\rangle$ away from the threshold; hence, a larger negative fluctuation is needed for a first down crossing, and, consequently, the lifetimes of the turbulent states will grow. Thus, there is a qualitative agreement with the results in Fig. 2.
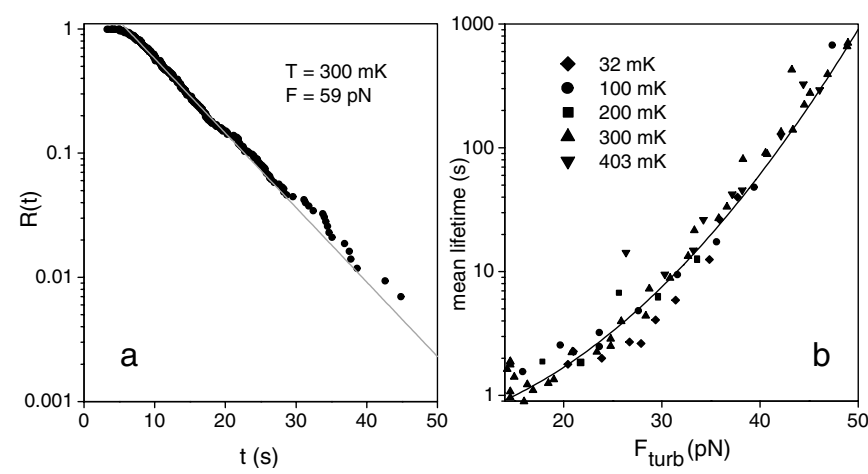

FIG. 2. Statistical analysis of the lifetimes $t$ of the turbulent phases. (a) The normalized numbers of turbulent phases $R(t)$ have an exponential distribution of $t$ at a given temperature and driving force. From the slope of the straight line fit the mean lifetime is obtained. (b) The mean lifetimes measured at different temperatures and driving forces as a function of the turbulent drag force. The solid line is a fit of Eq. (1).

For a more quantitative analysis we apply Rice's formula [6] for the average number $\langle N\rangle$ of down crossings per unit time of a level $C$ (below the mean value) of a stationary normal process $\xi(t)$ with zero mean and variance $\sigma_{\xi}^{2}$, namely,

$$
\langle N\rangle=\frac{1}{2 \pi} \sqrt{-r^{\prime \prime}(0)} \exp \left(-\frac{C^{2}}{2 \sigma_{\xi}^{2}}\right),
$$

where $r^{\prime \prime}(0)$ is the second derivative of the autocorrelation function of the random process at the origin and is related to the second spectral moment by

$$
-r^{\prime \prime}(0)=\int_{0}^{\infty} \omega^{2} S(\omega) d \omega
$$

with $S(\omega)$ being the spectral density function of the process. The exponential dependence of $\langle N\rangle$ on the level $C$ reflects the assumed normal probability density function (PDF) of the underlying process $f(\xi)=$ $\left(1 / \sqrt{2 \pi} \sigma_{\xi}\right) \exp \left(-\xi^{2} / 2 \sigma_{\xi}^{2}\right)$. From the measurements of the average time $\langle t\rangle$ of the first down crossing we have the condition

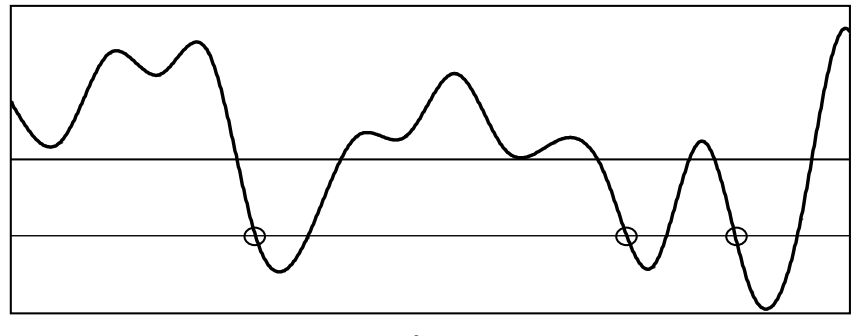

time

FIG. 3. Sketch of a statistical fluctuation of the vorticity with the down crossings of a given level below the mean value. The time of the first down crossing is the measured lifetime of a turbulent phase. 


$$
\langle N\rangle \cdot\langle t\rangle=1
$$

and therefore

$$
\langle t\rangle=\frac{1}{\langle N\rangle}=\frac{2 \pi}{\sqrt{-r^{\prime \prime}(0)}} \exp \left(\frac{C^{2}}{2 \sigma_{\xi}^{2}}\right) .
$$

Comparing Eq. (5) with Eq. (2) we can draw the following conclusions. $L^{2}$ fluctuates around the mean level $\left\langle L^{2}\right\rangle$, which is proportional to the dissipated power, and the down crossings refer to a level at zero vorticity. When a fluctuation reaches the $\left\langle L^{2}\right\rangle=0$ level, turbulence vanishes, of course, and a laminar phase is established temporarily. Furthermore, the fluctuations of $L^{2}$ have a normal distribution [7] with a standard deviation $\sigma_{L}=$ $L_{0}^{2} / \sqrt{2}=2.9 \times 10^{14} \mathrm{~m}^{-4}$. The typical length scale of this turbulent state is $L_{0}^{-1 / 2}=0.22 \mathrm{~mm}$, i.e., about the diameter of the sphere. This indicates a rather sparse vortex density, but one should keep in mind that this estimate is based purely on dimensional arguments and is valid probably only within an order of magnitude.

The spectral properties of the fluctuations of $L^{2}$ can be found from the second spectral moment. From Eqs. (5) and (2) we have $2 \pi / \sqrt{-r^{\prime \prime}(0)}=t_{0}=0.5 \mathrm{~s}$. For spectral densities that differ from zero only near $\omega=$ 0 , one can estimate the bandwidth (within a factor of order one [8]) $\Delta \omega \sim \sqrt{-r^{\prime \prime}(0)}=2 \pi / 0.5 \mathrm{~s}=13 \mathrm{~s}^{-1}$. This small value of the spectral bandwidth may be surprising, but from the preceding dimensional arguments we obtain the same typical time scale $\sim 1 / \kappa L_{0}=0.5 \mathrm{~s}$.

In summary, the finite lifetime of the turbulent flow of superfluid ${ }^{4} \mathrm{He}$ below $0.5 \mathrm{~K}$ around an oscillating sphere gives quantitative information on the properties of turbulent fluctuations of $L^{2}$, which is the square of the vorticity or the enstrophy in classical turbulence. The PDF of the fluctuations is shown to be of the Gaussian form, and the standard deviation can be obtained. From the measured second spectral moment the bandwidth can be estimated. Stability of turbulence is reached when the dissipated power and, hence, $\left\langle L^{2}\right\rangle$ is increased so much that the lifetime begins to exceed the measuring time. These results are found to be independent of temperature when thermally excited quasiparticles are negligible. It will be interesting to see how this behavior looks like in superfluid ${ }^{3} \mathrm{He}$. Finally, one could imagine that the model presented here is not only applicable to vorticity fluctuations in superfluid turbulence but to other instabilities as well, which are caused by random fluctuations exceeding a certain level.

The data that are analyzed here were obtained with the assistance of my students $\mathrm{H}$. Kerscher and M. Niemetz to whom I owe many thanks for a superb performance. An interesting discussion with $\mathrm{O}$. Avenel on Poisson processes is gratefully acknowledged.

[1] R. J. Donnelly, Quantized Vortices in Helium II (Cambridge University Press, Cambridge, 1991).

[2] Quantized Vortex Dynamics and Superfluid Turbulence, edited by C. F. Barenghi, R. J. Donnelly, and W. F. Vinen (Springer, Berlin, 2001).

[3] A. P. Finne, T. Araki, R. Blaauwgeers, V. B. Eltsov, N. B. Kopnin, M. Krusius, L. Skrbek, M. Tsubota, and G. E. Volovik, Nature (London) 424, 1022 (2003).

[4] M. Niemetz, H. Kerscher, and W. Schoepe, in Quantized Vortex Dynamics and Superfluid Turbulence, edited by C. F. Barenghi, R. J. Donnelly, and W. F. Vinen (Springer, Berlin, 2001), p. 87; M. Niemetz, H. Kerscher, and W. Schoepe, J. Low Temp. Phys. 126, 287 (2002); M. Niemetz and W. Schoepe (to be published).

[5] W. F. Vinen and J. J. Niemela, J. Low Temp. Phys. 128, 167 (2002).

[6] M. R. Leadbetter, Georg Lindgren, and Holger Rootzén, Extremes and Related Properties of Random Sequences and Processes (Springer-Verlag, New York, 1983).

[7] To infer the PDF of the process from $\langle N\rangle$ is usually (but not necessarily) possible. Exact conditions are given in Refs. [6,8].

[8] V. I. Tikhonov, Usp. Fiz. Nauk 77, 449 (1962) [Sov. Phys. Usp. 5, 594 (1963)]. 